\title{
Complexity of Proper Prefix-Convex Regular Languages ${ }^{\star}$
}

\author{
Janusz A. Brzozowski and Corwin Sinnamon \\ David R. Cheriton School of Computer Science, University of Waterloo, \\ Waterloo, ON, Canada N2L 3G1 \\ brzozo@uwaterloo.ca, sinncore@gmail.com
}

\begin{abstract}
A language $L$ over an alphabet $\Sigma$ is prefix-convex if, for any words $x, y, z \in \Sigma^{*}$, whenever $x$ and $x y z$ are in $L$, then so is $x y$. Prefix-convex languages include right-ideal, prefix-closed, and prefix-free languages, which were studied elsewhere. Here we concentrate on prefixconvex languages that do not belong to any one of these classes; we call such languages proper. We exhibit most complex proper prefix-convex languages, which meet the bounds for the size of the syntactic semigroup, reversal, complexity of atoms, star, product, and boolean operations.
\end{abstract}

Keywords: atom, most complex, prefix-convex, proper, quotient complexity, regular language, state complexity, syntactic semigroup

\section{Introduction}

Prefix-Convex Languages We examine the complexity properties of a class of regular languages that has never been studied before: the class of proper prefixconvex languages [7]. Let $\Sigma$ be a finite alphabet; if $w=x y$, for $x, y \in \Sigma^{*}$, then $x$ is a prefix of $w$. A language $L \subseteq \Sigma^{*}$ is prefix-convex $[1,16]$ if whenever $x$ and $x y z$ are in $L$, then so is $x y$. Prefix-convex languages include three special cases:

1. A language $L \subseteq \Sigma$ is a right ideal if it is non-empty and satisfies $L=L \Sigma^{*}$. Right ideals appear in pattern matching [11]: $L \Sigma^{*}$ is the set of all words in some text (word in $\Sigma^{*}$ ) beginning with words in $L$.

2. A language is prefix-closed [6] if whenever $w$ is in $L$, then so is every prefix of $w$. The set of allowed sequences to any system is prefix-closed. Every prefix-closed language other than $\Sigma^{*}$ is the complement of a right ideal [1].

3. A language is prefix-free if $w \in L$ implies that no prefix of $w$ other than $w$ is in $L$. Prefix-free languages other than $\{\varepsilon\}$, where $\varepsilon$ is the empty word, are prefix codes and are of considerable importance in coding theory [2].

The complexities of these three special prefix-convex languages were studied in [8]. We now turn to the "real" prefix-convex languages that do not belong to any of the three special classes.

Omitted proofs can be found in [7].

\footnotetext{
* This work was supported by the Natural Sciences and Engineering Research Council of Canada grant No. OGP0000871.
} 
Complexities of Operations If $L \subseteq \Sigma^{*}$ is a language, the (left) quotient of $L$ by a word $w \in \Sigma^{*}$ is $w^{-1} L=\{x \mid w x \in L\}$. A language is regular if and only if it has a finite number of distinct quotients. So the number of quotients of $L$, the quotient complexity [3] $\kappa(L)$ of $L$, is a natural measure of complexity for $L$. An equivalent concept is the state complexity $[15,17,18]$ of $L$, which is the number of states in a complete minimal deterministic finite automaton (DFA) over $\Sigma$ recognizing $L$. We refer to quotient/state complexity simply as complexity.

If $L_{n}$ is a regular language of complexity $n$, and $\circ$ is a unary operation, the complexity of $\circ$ is the maximal value of $\kappa\left(L_{n}^{\circ}\right)$, expressed as a function of $n$, as $L_{n}$ ranges over all languages of complexity $n$. If $L_{m}^{\prime}$ and $L_{n}$ are regular languages of complexities $m$ and $n$ respectively, and $\circ$ is a binary operation, the complexity of $\circ$ is the maximal value of $\kappa\left(L_{m}^{\prime} \circ L_{n}\right)$, expressed as a function of $m$ and $n$, as $L_{m}^{\prime}$ and $L_{n}$ range over all languages of complexities $m$ and $n$. The complexity of an operation is a lower bound on its time and space complexities. The operations reversal, (Kleene) star, product (concatenation), and binary boolean operations are considered "common", and their complexities are known; see $[4,17,18]$.

Witnesses To find the complexity of a unary operation we find an upper bound on this complexity, and languages that meet this bound. We require a language $L_{n}$ for each $n$, that is, a sequence, $\left(L_{k}, L_{k+1}, \ldots\right)$, called a stream of languages, where $k$ is a small integer, because the bound may not hold for small values of $n$. For a binary operation we need two streams. The same stream cannot always be used for both operands, but for all common binary operations the second stream can be a "dialect" of the first, that is it can "differ only slightly" from the first [4]. Let $\Sigma=\left\{a_{1}, \ldots, a_{k}\right\}$ be an alphabet ordered as shown; if $L \subseteq \Sigma^{*}$, we denote it by $L\left(a_{1}, \ldots, a_{k}\right)$. A dialect of $L$ is obtained by deleting letters of $\Sigma$ in the words of $L$, or replacing them by letters of another alphabet $\Sigma^{\prime}$. More precisely, for an injective partial map $\pi: \Sigma \mapsto \Sigma^{\prime}$, we get a dialect of $L$ by replacing each letter $a \in \Sigma$ by $\pi(a)$ in every word of $L$, or deleting the word if $\pi(a)$ is undefined. We write $L\left(\pi\left(a_{1}\right), \ldots, \pi\left(a_{k}\right)\right)$ to denote the dialect of $L\left(a_{1}, \ldots, a_{k}\right)$ given by $\pi$, and we denote undefined values of $\pi$ by "-". Undefined values for letters at the end of the alphabet are omitted; for example, $L(a, c,-,-)$ is written as $L(a, c)$. Our definition of dialect is more general than that of [5], where only the case $\Sigma^{\prime}=\Sigma$ was allowed.

Finite Automata A deterministic finite automaton (DFA) is a quintuple $\mathcal{D}=$ $\left(Q, \Sigma, \delta, q_{0}, F\right)$, where $Q$ is a finite non-empty set of states, $\Sigma$ is a finite nonempty alphabet, $\delta: Q \times \Sigma \rightarrow Q$ is the transition function, $q_{0} \in Q$ is the initial state, and $F \subseteq Q$ is the set of final states. We extend $\delta$ to a function $\delta: Q \times \Sigma^{*} \rightarrow$ $Q$ as usual. A DFA $\mathcal{D}$ accepts a word $w \in \Sigma^{*}$ if $\delta\left(q_{0}, w\right) \in F$. The set of all words accepted by $\mathcal{D}$ is the language of $\mathcal{D}$. If $q \in Q$, then the language $L_{q}$ of $q$ is the language accepted by the DFA $(Q, \Sigma, \delta, q, F)$. A state is empty or dead or a sink if its language is empty. Two states $p$ and $q$ of $\mathcal{D}$ are equivalent if $L_{p}=L_{q}$. A state $q$ is reachable if there exists $w \in \Sigma^{*}$ such that $\delta\left(q_{0}, w\right)=q$. A DFA is minimal if all of its states are reachable and no two states are equivalent. A nondeterministic finite automaton (NFA) is a quintuple $\mathcal{D}=(Q, \Sigma, \delta, I, F)$, where $Q, \Sigma$ and $F$ are defined as in a DFA, $\delta: Q \times \Sigma \rightarrow 2^{Q}$ is the transition 
function, and $I \subseteq Q$ is the set of initial states. An $\varepsilon-N F A$ is an NFA in which transitions under the empty word $\varepsilon$ are also permitted.

Transformations We use $Q_{n}=\{0, \ldots, n-1\}$ as the set of states of every DFA with $n$ states. A transformation of $Q_{n}$ is a mapping $t: Q_{n} \rightarrow Q_{n}$. The image of $q \in Q_{n}$ under $t$ is $q t$. In any DFA, each letter $a \in \Sigma$ induces a transformation $\delta_{a}$ of the set $Q_{n}$ defined by $q \delta_{a}=\delta(q, a)$; we denote this by $a: \delta_{a}$. Often we use the letter $a$ to denote the transformation it induces; thus we write $q a$ instead of $q \delta_{a}$. We extend the notation to sets: if $P \subseteq Q_{n}$, then $P a=\{p a \mid p \in P\}$. We also write $P \stackrel{a}{\longrightarrow} P a$ to indicate that the image of $P$ under $a$ is $P a$. If $s, t$ are transformations of $Q_{n}$, their composition is (qs)t.

For $k \geqslant 2$, a transformation (permutation) $t$ of a set $P=\left\{q_{0}, q_{1}, \ldots, q_{k-1}\right\} \subseteq$ $Q_{n}$ is a $k$-cycle if $q_{0} t=q_{1}, q_{1} t=q_{2}, \ldots, q_{k-2} t=q_{k-1}, q_{k-1} t=q_{0}$. This $k$-cycle is denoted by $\left(q_{0}, q_{1}, \ldots, q_{k-1}\right)$. A 2 -cycle $\left(q_{0}, q_{1}\right)$ is called a transposition. A transformation that sends all the states of $P$ to $q$ and acts as the identity on the other states is denoted by $(P \rightarrow q)$, and $\left(Q_{n} \rightarrow p\right)$ is called a constant transformation. If $P=\{p\}$ we write $(p \rightarrow q)$ for $(\{p\} \rightarrow q)$. The identity transformation is denoted by $\mathbb{1}$. Also, $\left({ }_{i}^{j} q \rightarrow q+1\right)$ is a transformation that sends $q$ to $q+1$ for $i \leqslant q \leqslant j$ and is the identity for the remaining states; $\left({ }_{i}^{j} q \rightarrow q-1\right)$ is defined similarly.

Semigroups The syntactic congruence of $L \subseteq \Sigma^{*}$ is defined on $\Sigma^{+}$: For $x, y \in$ $\Sigma^{+}, x \approx_{L} y$ if and only if $w x z \in L \Leftrightarrow w y z \in L$ for all $w, z \in \Sigma^{*}$. The quotient set $\Sigma^{+} / \approx_{L}$ of equivalence classes of $\approx_{L}$ is the syntactic semigroup of $L$. Let $\mathcal{D}_{n}=\left(Q_{n}, \Sigma, \delta, q_{0}, F\right)$ be a DFA, and let $L_{n}=L\left(\mathcal{D}_{n}\right)$. For each word $w \in \Sigma^{*}$, the transition function induces a transformation $\delta_{w}$ of $Q_{n}$ by $w$ : for all $q \in Q_{n}$, $q \delta_{w}=\delta(q, w)$. The set $T_{\mathcal{D}_{n}}$ of all such transformations by non-empty words is a semigroup under composition called the transition semigroup of $\mathcal{D}_{n}$. If $\mathcal{D}_{n}$ is a minimal DFA of $L_{n}$, then $T_{\mathcal{D}_{n}}$ is isomorphic to the syntactic semigroup $T_{L_{n}}$ of $L_{n}$, and we represent elements of $T_{L_{n}}$ by transformations in $T_{\mathcal{D}_{n}}$. The size of the syntactic semigroup has been used as a measure of complexity for regular languages $[4,10,12,14]$.

Atoms are defined by a left congruence, where two words $x$ and $y$ are equivalent if $u x \in L$ if and only if $u y \in L$ for all $u \in \Sigma^{*}$. Thus $x$ and $y$ are equivalent if $x \in u^{-1} L$ if and only if $y \in u^{-1} L$. An equivalence class of this relation is an atom of $L[9,13]$.

One can conclude that an atom is a non-empty intersection of complemented and uncomplemented quotients of $L$. That is, every atom of a language with quotients $K_{0}, K_{1}, \ldots, K_{n-1}$ can be written as $A_{S}=\bigcap_{i \in S} K_{i} \cap \bigcap_{i \in \bar{S}} \overline{K_{i}}$ for some set $S \subseteq Q_{n}$. The number of atoms and their complexities were suggested as possible measures of complexity [4], because all the quotients of a language and the quotients of its atoms are unions of atoms [9].

Most Complex Regular Stream The stream $\left(\mathcal{D}_{n}(a, b, c) \mid n \geqslant 3\right)$ of Definition 1 and Figure 1 will be used as a component in the class of proper prefixconvex languages. This stream together with some dialects meets the complexity bounds for reversal, star, product, and all binary boolean operations [7, 8]. More- 
over, it has the maximal syntactic semigroup and most complex atoms, making it a most complex regular stream.

Definition 1. For $n \geqslant 3$, let $\mathcal{D}_{n}=\mathcal{D}_{n}(a, b, c)=\left(Q_{n}, \Sigma, \delta_{n}, 0,\{n-1\}\right)$, where $\Sigma=\{a, b, c\}$, and $\delta_{n}$ is defined by $a:(0, \ldots, n-1), b:(0,1), c:(1 \rightarrow 0)$.

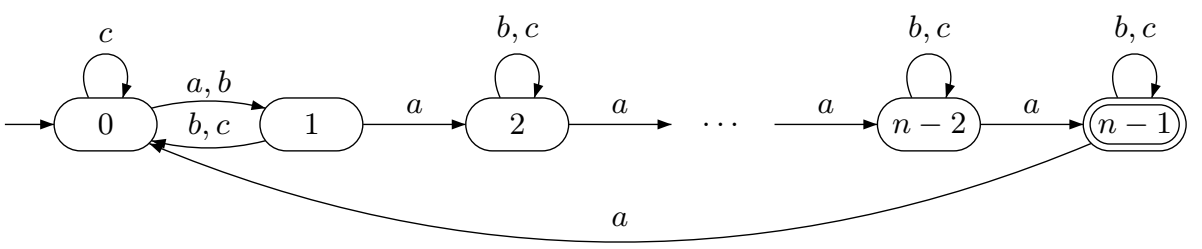

Fig. 1. Minimal DFA of a most complex regular language.

Most complex streams are useful in systems dealing with regular languages and finite automata. To know the maximal sizes of automata that can be handled by a system it suffices to use the most complex stream to test all the operations.

\section{Proper Prefix-Convex Languages}

We begin with some properties of prefix-convex languages that will be used frequently in this section. The following lemma and propositions characterize the classes of prefix-convex languages in terms of their minimal DFAs.

Lemma 1. Let $L$ be a prefix-convex language over $\Sigma$. Either $L$ is a right ideal or $L$ has an empty quotient.

Proposition 1. Let $L_{n}$ be a regular language of complexity $n$, and let $\mathcal{D}_{n}=$ $\left(Q_{n}, \Sigma, \delta, 0, F\right)$ be a minimal DFA recognizing $L_{n}$. The following are equivalent:

1. $L_{n}$ is prefix-convex.

2. For all $p, q, r \in Q_{n}$, if $p$ and $r$ are final, $q$ is reachable from $p$, and $r$ is reachable from $q$, then $q$ is final.

3. Every state reachable in $\mathcal{D}_{n}$ from any final state is either final or empty.

Proposition 2. Let $L_{n}$ be a non-empty prefix-convex language of complexity $n$, and let $\mathcal{D}_{n}=\left(Q_{n}, \Sigma, \delta, 0, F\right)$ be a minimal DFA recognizing $L_{n}$.

1. $L_{n}$ is prefix-closed if and only if $0 \in F$.

2. $L_{n}$ is prefix-free if and only if $\mathcal{D}_{n}$ has a unique final state $p$ and an empty state $p^{\prime}$ such that $\delta(p, a)=p^{\prime}$ for all $a \in \Sigma$.

3. $L_{n}$ is a right ideal if and only if $\mathcal{D}_{n}$ has a unique final state $p$ and $\delta(p, a)=p$ for all $a \in \Sigma$. 
A prefix-convex language $L$ is proper if it is not a right ideal and it is neither prefix-closed nor prefix-free. We say it is $k$-proper if it has $k$ final states, $1 \leqslant$ $k \leqslant n-2$. Every minimal DFA for a $k$-proper language with complexity $n$ has the same general structure: there are $n-1-k$ non-final, non-empty states, $k$ final states, and one empty state. Every letter fixes the empty state and, by Proposition 1, no letter sends a final state to a non-final, non-empty state.

Next we define a stream of $k$-proper DFAs and languages, which we will show to be most complex.

Definition 2. For $n \geqslant 3,1 \leqslant k \leqslant n-2$, let $\mathcal{D}_{n, k}(\Sigma)=\left(Q_{n}, \Sigma, \delta_{n, k}, 0, F_{n, k}\right)$ where $\Sigma=\left\{a, b, c_{1}, c_{2}, d_{1}, d_{2}, e\right\}, F_{n, k}=\{n-1-k, \ldots, n-2\}$, and $\delta_{n, k}$ is given by the transformations

$$
\begin{aligned}
& a: \begin{cases}(1, \ldots, n-2-k)(n-1-k, n-k), & \text { if } n-1-k \text { is even and } k \geqslant 2 ; \\
(0, \ldots, n-2-k)(n-1-k, n-k), & \text { if } n-1-k \text { is odd and } k \geqslant 2 ; \\
(1, \ldots, n-2-k), & \text { if } n-1-k \text { is even and } k=1 ; \\
(0, \ldots, n-2-k), & \text { if } n-1-k \text { is odd and } k=1 .\end{cases} \\
& b: \begin{cases}(n-k, \ldots, n-2)(0,1), & \text { if } k \text { is even and } n-1-k \geqslant 2 ; \\
(n-1-k, \ldots, n-2)(0,1), & \text { if } k \text { is odd and } n-1-k \geqslant 2 ; \\
(n-1-k, \ldots, n-2), & \text { if } k \text { is even and } n-1-k=1 ;\end{cases} \\
& c_{1}:\left\{\begin{array}{c}
(1-0), \quad \text { if } n-1-k \geqslant 2 ; \\
\mathbb{1}, \quad \text { if } n-1-k=1 .
\end{array}\right. \\
& c_{2}:\left\{\begin{array}{c}
(n-k \rightarrow n-1-k), \quad \text { if } k \geqslant 2 ; \\
d_{1}:
\end{array}\right. \\
& d_{2}:(n-2-k \rightarrow n-1)\left(\begin{array}{l}
n-3-k \\
n-1-k
\end{array} \rightarrow q \rightarrow q+1\right) .
\end{aligned}
$$

Also, let $E_{n, k}=\{0, \ldots, n-2-k\}$; it is useful to partition $Q_{n}$ into $E_{n, k}, F_{n, k}$, and $\{n-1\}$. Letters $a$ and $b$ have complementary behaviours on $E_{n, k}$ and $F_{n, k}$, depending on the parities of $n$ and $k$. Letters $c_{1}$ and $d_{1}$ act on $E_{n, k}$ in exactly the same way as $c_{2}$ and $d_{2}$ act on $F_{n, k}$. In addition, $d_{1}$ and $d_{2}$ send states $n-2-k$ and $n-2$, respectively, to state $n-1$, and letter $e$ connects the two parts of the $D F A$. The structure of $\mathcal{D}_{n}(\Sigma)$ is shown in Figures 2 and 3 for certain parities of $n-1-k$ and $k$. Let $L_{n, k}(\Sigma)$ be the language recognized by $\mathcal{D}_{n, k}(\Sigma)$.

Theorem 1 (Proper Prefix-Convex Languages). For $n \geqslant 3$ and $1 \leqslant k \leqslant$ $n-2$, the DFA $\mathcal{D}_{n, k}(\Sigma)$ of Definition 2 is minimal and $L_{n, k}(\Sigma)$ is a $k$-proper language of complexity $n$. The bounds below are maximal for $k$-proper prefixconvex languages. At least seven letters are required to meet these bounds. 


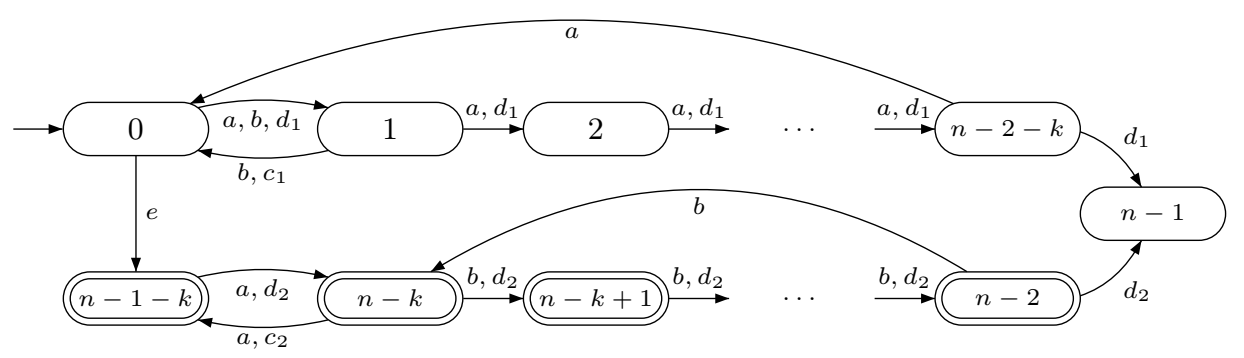

Fig. 2. DFA $\mathcal{D}_{n, k}\left(a, b, c_{1}, c_{2}, d_{1}, d_{2}, e\right)$ of Definition 2 when $n-1-k$ is odd, $k$ is even, and both are at least 2 ; missing transitions are self-loops.

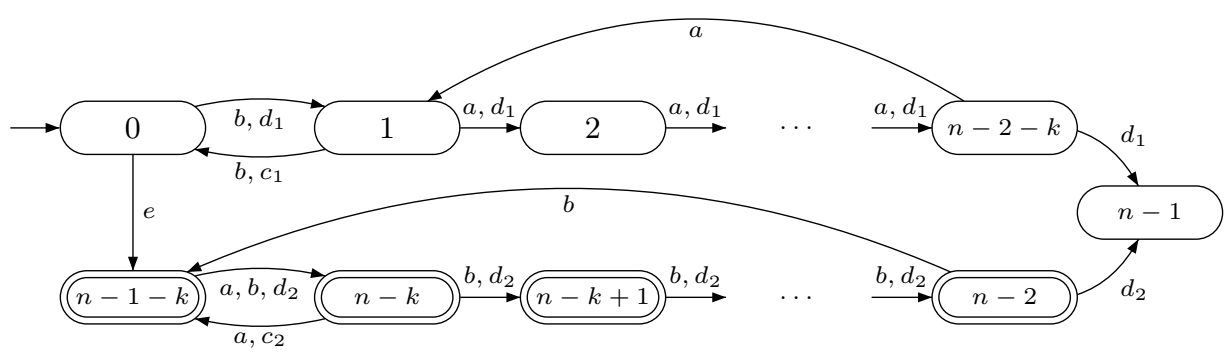

Fig. 3. DFA $\mathcal{D}_{n, k}\left(a, b, c_{1}, c_{2}, d_{1}, d_{2}, e\right)$ of Definition 2 when $n-1-k$ is even, $k$ is odd, and both are at least 2 ; missing transitions are self-loops.

1. The syntactic semigroup of $L_{n, k}(\Sigma)$ has cardinality $n^{n-1-k}(k+1)^{k}$; the maximal value $n(n-1)^{n-2}$ is reached only when $k=n-2$.

2. The non-empty, non-final quotients of $L_{n, k}\left(a, b,-,-,-, d_{2}, e\right)$ have complexity $n$, the final quotients have complexity $k+1$, and $\emptyset$ has complexity 1.

3. The reverse of $L_{n, k}\left(a, b,-,-,-, d_{2}, e\right)$ has complexity $2^{n-1}$; moreover, the language $L_{n, k}\left(a, b,-,-,-, d_{2}, e\right)$ has $2^{n-1}$ atoms for all $k$.

4. For each atom $A_{S}$ of $L_{n, k}(\Sigma)$, write $S=X_{1} \cup X_{2}$, where $X_{1} \subseteq E_{n, k}$ and $X_{2} \subseteq F_{n, k}$. Let $\overline{X_{1}}=E_{n, k} \backslash X_{1}$ and $\overline{X_{2}}=F_{n, k} \backslash X_{2}$. If $X_{2} \neq \emptyset$, then $\kappa\left(A_{S}\right)=$

$$
1+\sum_{x_{1}=0}^{\left|X_{1}\right|} \sum_{x_{2}=1}^{\left|X_{1}\right|+\left|X_{2}\right|-x_{1}} \sum_{y_{1}=0}^{\left|\overline{X_{1}}\right|} \sum_{y_{2}=0}^{\left|\overline{X_{1}}\right|+\left|\overline{X_{2}}\right|-y_{1}}\left(\begin{array}{c}
n-1-k \\
x_{1}
\end{array}\right)\left(\begin{array}{c}
k \\
x_{2}
\end{array}\right)\left(\begin{array}{c}
n-1-k-x_{1} \\
y_{1}
\end{array}\right)\left(\begin{array}{c}
k-x_{2} \\
y_{2}
\end{array}\right) .
$$

If $X_{1} \neq \emptyset$ and $X_{2}=\emptyset$, then $\kappa\left(A_{S}\right)=$

$1+\sum_{x_{1}=0}^{\left|X_{1}\right|} \sum_{x_{2}=0}^{\left|X_{1}\right|-x_{1}} \sum_{y_{1}=0}^{\left|\overline{X_{1}}\right|} \sum_{y_{2}=0}^{k}\left(\begin{array}{c}n-1-k \\ x_{1}\end{array}\right)\left(\begin{array}{c}k \\ x_{2}\end{array}\right)\left(\begin{array}{c}n-1-k-x_{1} \\ y_{1}\end{array}\right)\left(\begin{array}{c}k-x_{2} \\ y_{2}\end{array}\right)-2^{k} \sum_{y=0}^{\left|\overline{X x_{1}}\right|}\left(\begin{array}{c}n-1-k \\ y\end{array}\right)$.

Otherwise, $S=\emptyset$ and $\kappa\left(A_{S}\right)=2^{n-1}$.

5. The star of $L_{n, k}\left(a, b,-,-, d_{1}, d_{2}, e\right)$ has complexity $2^{n-2}+2^{n-2-k}+1$. The maximal value $2^{n-2}+2^{n-3}+1$ is reached only when $k=1$. 
6. $L_{m, j}^{\prime}\left(a, b, c_{1},-, d_{1}, d_{2}, e\right) L_{n, k}\left(a, d_{2}, c_{1},-, d_{1}, b, e\right)$ has complexity $m-1-j+$ $j 2^{n-2}+2^{n-1}$. The maximal value $m 2^{n-2}+1$ is reached only when $j=m-2$.

7. For $m, n \geqslant 3,1 \leqslant j \leqslant m-2$, and $1 \leqslant k \leqslant n-2$, define the languages $L_{m, j}^{\prime}=L_{m, j}^{\prime}\left(a, b, c_{1},-, d_{1}, d_{2}, e\right)$ and $L_{n, k}=L_{n, k}\left(a, b, e,-, d_{2}, d_{1}, c_{1}\right)$. For any proper binary boolean function $\circ$, the complexity of $L_{m, j}^{\prime} \circ L_{n, k}$ is maximal. In particular,

(a) $L_{m, j}^{\prime} \cup L_{n, k}$ and $L_{m, j}^{\prime} \oplus L_{n, k}$ have complexity $m n$.

(b) $L_{m, j}^{\prime} \backslash L_{n, k}$ has complexity $m n-(n-1)$.

(c) $L_{m, j}^{\prime} \cap L_{n, k}$ has complexity $m n-(m+n-2)$.

Proof. The remainder of this paper is an outline of the proof of this theorem. The longer parts of the proof are separated into individual propositions and lemmas.

DFA $\mathcal{D}_{n, k}\left(a, b,-,-,-, d_{2}, e\right)$ is easily seen to be minimal. Language $L_{n, k}(\Sigma)$ is $k$-proper by Propositions 1 and 2 .

1. See Lemma 2 and Proposition 3.

2. If the initial state of $\mathcal{D}_{n, k}\left(a, b,-,-,-, d_{2}, e\right)$ is changed to $q \in E_{n, k}$, the new DFA accepts a quotient of $L_{n, k}$ and is still minimal; hence the complexity of that quotient is $n$. If the initial state is changed to $q \in F_{n, k}$ then states in $E_{n, k}$ are unreachable, but the DFA on $\{n-1-k, \ldots, n-1\}$ is minimal; hence the complexity of that quotient is $k+1$. The remaining quotient is empty, and hence has complexity 1. By Proposition 1, these are maximal.

3. See Proposition 4 for the reverse. It was shown in [9] that the number of atoms is equal to the complexity of the reverse.

4. See [7].

5. See Proposition 5.

6. See [7].

7. By [3, Theorem 2], all boolean operations on regular languages have the upper bound $m n$, which gives the bound for (a). The bounds for (b) and (c) follow from [3, Theorem 5]. The proof that all these bounds are tight for $L_{m, j}^{\prime} \circ L_{n, k}$ can be found in [7].

Lemma 2. Let $n \geqslant 1$ and $1 \leqslant k \leqslant n-2$. For any permutation $t$ of $Q_{n}$ such that $E_{n, k} t=E_{n, k}, F_{n, k} t=F_{n, k}$, and $(n-1) t=n-1$, there is a word $w \in\{a, b\}^{*}$ that induces $t$ on $\mathcal{D}_{n, k}$.

Proof. Only $a$ and $b$ induce permutations of $Q_{n}$; every other letter induces a properly injective map. Furthermore, $a$ and $b$ permute $E_{n, k}$ and $F_{n, k}$ separately, and both fix $n-1$. Hence every $w \in\{a, b\}^{*}$ induces a permutation on $Q_{n}$ such that $E_{n, k} w=E_{n, k}, F_{n, k} w=F_{n, k}$, and $(n-1) w=n-1$. Each such permutation naturally corresponds to an element of $S_{n-1-k} \times S_{k}$, where $S_{m}$ denotes the symmetric group on $m$ elements. To be consistent with the DFA, assume $S_{n-1-k}$ contains permutations of $\{0, \ldots, n-2-k\}$ and $S_{k}$ contains permutations of $\{n-1-k, \ldots, n-2\}$. Let $s_{a}$ and $s_{b}$ denote the group elements corresponding to the transformations induced by $a$ and $b$ respectively. We show that $s_{a}$ and $s_{b}$ generate $S_{n-1-k} \times S_{k}$. 
It is well known that $(0, \ldots, m-1)$, and $(0,1)$ generate the symmetric group on $\{0, \ldots, m-1\}$ for any $m \geq 2$. Note that $(1, \ldots, m-1)$ and $(0,1)$ are also generators, since $(0,1)(1, \ldots, m-1)=(0, \ldots, m-1)$.

If $n-1-k=1$ and $k=1$, then $S_{n-1-k} \times S_{k}$ is the trivial group. If $n-1-k=1$ and $k \geqslant 2$, then $s_{a}=(\mathbb{1},(n-1-k, n-k))$ and $s_{b}$ is either $(\mathbb{1},(n-1-k, \ldots, n-2))$ or $(\mathbb{1},(n-k, \ldots, n-2))$, and either pair generates the group. There is a similar argument when $k=1$.

Assume now $n-1-k \geqslant 2$ and $k \geqslant 2$. If $n-1-k$ is odd then $s_{a}=((0, \ldots, n-$ $2-k),(n-1-k, n-k))$, and hence $s_{a}^{n-1-k}=\left((0, \ldots, n-2-k)^{n-1-k},(n-\right.$ $\left.1-k, n-k)^{n-1-k}\right)=(\mathbb{1},(n-1-k, n-k))$. Similarly if $n-1-k$ is even then $s_{a}=((1, \ldots, n-2-k),(n-1-k, n-k))$, and hence $s_{a}^{n-2-k}=(\mathbb{1},(n-1-k, n-k))$. Therefore $(\mathbb{1},(n-1-k, n-k))$ is always generated by $s_{a}$. By symmetry, $((0,1), \mathbb{1})$ is always generated by $s_{b}$ regardless of the parity of $k$.

Since we can isolate the transposition component of $s_{a}$, we can isolate the other component as well: $(\mathbb{1},(n-1-k, n-k)) s_{a}$ is either $((0, \ldots, n-2-k), \mathbb{1})$ or $((1, \ldots, n-2-k), \mathbb{1})$. Paired with $((0,1), \mathbb{1})$, either element is sufficient to generate $S_{n-1-k} \times\{\mathbb{1}\}$. Similarly, $s_{a}$ and $s_{b}$ generate $\{\mathbb{1}\} \times S_{k}$. Therefore $s_{a}$ and $s_{b}$ generate $S_{n-1-k} \times S_{k}$. It follows that $a$ and $b$ generate all permutations $t$ of $Q_{n}$ such that $E_{n, k} t=E_{n, k}, F_{n, k} t=F_{n, k}$, and $(n-1) t=n-1$.

Proposition 3 (Syntactic Semigroup). The syntactic semigroup of $L_{n, k}(\Sigma)$ has cardinality $n^{n-1-k}(k+1)^{k}$, which is maximal for a $k$-proper language. Furthermore, seven letters are required to meet this bound. The maximum value $n(n-1)^{n-2}$ is reached only when $k=n-2$.

Proof. Let $L$ be a $k$-proper language of complexity $n$ and let $\mathcal{D}$ be a minimal DFA recognizing $L$. By Lemma $1, \mathcal{D}$ has an empty state. By Proposition 1 , the only states that can be reached from one of the $k$ final states are either final or empty. Thus, a transformation in the transition semigroup of $\mathcal{D}$ may map each final state to one of $k+1$ possible states, while each non-final, non-empty state may be mapped to any of the $n$ states. Since the empty state can only be mapped to itself, we are left with $n^{n-1-k}(k+1)^{k}$ possible transformations in the transition semigroup. Therefore the syntactic semigroup of any $k$-proper language has size at most $n^{n-1-k}(k+1)^{k}$.

Now consider the transition semigroup of $\mathcal{D}_{n, k}(\Sigma)$. Every transformation $t$ in the semigroup must satisfy $F_{n, k} t \subseteq F_{n, k} \cup\{n-1\}$ and $(n-1) t=n-1$, since any other transformation would violate prefix-convexity. We show that the semigroup contains every such transformation, and hence the syntactic semigroup of $L_{n, k}(\Sigma)$ is maximal.

First, consider the transformations $t$ such that $E_{n, k} t \subseteq E_{n, k} \cup\{n-1\}$ and $q t=q$ for all $q \in F_{n, k} \cup\{n-1\}$. By Lemma $2, a$ and $b$ generate every permutation of $E_{n, k}$. When $t$ is not a permutation, we can use $c_{1}$ to combine any states $p$ and $q$ : apply a permutation on $E_{n, k}$ so that $p \rightarrow 0$ and $q \rightarrow 1$, and then apply $c_{1}$ so that $1 \rightarrow 0$. Repeat this method to combine any set of states, and further apply permutations to induce the desired transformation while leaving the states of $F_{n, k} \cup\{n-1\}$ in place. The same idea applies with $d_{1}$; apply permutations 
and $d_{1}$ to send any states of $E_{n, k}$ to $n-1$. Hence $a, b, c_{1}$, and $d_{1}$ generate every transformation $t$ such that $E_{n, k} t \subseteq E_{n, k} \cup\{n-1\}$ and $q t=q$ for all $q \in F_{n, k} \cup\{n-1\}$.

We can make the same argument for transformations that act only on $F_{n, k}$ and fix every other state. Since $c_{2}$ and $d_{2}$ act on $F_{n, k}$ exactly as $c_{1}$ and $d_{1}$ act on $E_{n, k}$, the letters $a, b, c_{2}$, and $d_{2}$ generate every transformation $t$ such that $F_{n, k} t \subseteq F_{n, k} \cup\{n-1\}$ and $q t=q$ for all $q \in E_{n, k} \cup\{n-1\}$. It follows that $a, b, c_{1}$, $c_{2}, d_{1}$, and $d_{2}$ generate every transformation $t$ such that $E_{n, k} t \subseteq E_{n, k} \cup\{n-1\}$, $F_{n, k} t \subseteq F_{n, k} \cup\{n-1\}$, and $(n-1) t=n-1$.

Note the similarity between this DFA restricted to the states $E_{n, k} \cup\{n-1\}$ (or $F_{n, k} \cup\{n-1\}$ ) and the witness for right ideals introduced in [7]. The argument for the size of the syntactic semigroup of right ideals is similar to this; see [10].

Finally, consider an arbitrary transformation $t$ such that $F_{n, k} t \subseteq F_{n, k} \cup\{n-1\}$ and $(n-1) t=n-1$. Let $j_{t}$ be the number of states $p \in E_{n, k}$ such that $p t \in F_{n, k}$. We show by induction on $j_{t}$ that $t$ is in the transition semigroup of $\mathcal{D}$. If $j_{t}=0$, then $t$ is generated by $\Sigma \backslash\{e\}$. If $j_{t} \geqslant 1$, there exist $p, q \in E_{n, k}$ such that $p t \in F_{n, k}$ and $q$ is not in the image of $t$. Consider the transformations $s_{1}$ and $s_{2}$ defined by $q s_{1}=p t$ and $r s_{1}=r$ for $r \neq q$, and $p s_{2}=q$ and $r s_{2}=r t$ for $r \neq p$. Then $\left(r s_{2}\right) s_{1}=r t$ for all $r \in Q_{n}$. Notice that $j_{s_{2}}=j_{t}-1$, and hence $\Sigma$ generates $s_{2}$ by inductive assumption. One can verify that $s_{1}=(n-$ $1-k, p t)(0, q)(0 \rightarrow n-1-k)(0, q)(n-1-k, p t)$. From this expression, we see that $s_{1}$ is the composition of transpositions induced by words in $\{a, b\}^{*}$ and the transformation $(0 \rightarrow n-1-k)$ induced by $e$, and hence $s_{1}$ is generated by $\Sigma$. Thus, $t$ is in the transition semigroup. By induction on $j_{t}$, it follows that the syntactic semigroup of $L_{n, k}$ is maximal.

Now we show that seven letters are required to meet this bound. Two letters (like $a$ and $b$ ) are required to generate the permutations, since clearly one letter is not sufficient. Every other letter will induce a properly injective map. A letter (like $c_{1}$ ) that induces a properly injective map on $E_{n, k}$ and permutes $F_{n, k}$ is required. Similarly, a letter (like $c_{2}$ ) that permutes $E_{n, k}$ and induces a properly injective map on $F_{n, k}$ is required. A letter (like $d_{1}$ ) that sends a state in $E_{n, k}$ to $n-1$ and permutes $F_{n, k}$ is required. Similarly, a letter (like $d_{2}$ ) that sends a state in $F_{n, k}$ to $n-1$ and permutes $E_{n, k}$ is required. Finally, a letter (like $e$ ) that connects $E_{n, k}$ and $F_{n, k}$ is required.

For a fixed $n$, we may want to know which $k \in\{1, \ldots, n-2\}$ maximizes $s_{n}(k)=n^{n-1-k}(k+1)^{k}$; this corresponds to the largest syntactic semigroup of a proper prefix-convex language with $n$ quotients. We show that $s_{n}(k)$ is largest at $k=n-2$. Consider the ratio $\frac{s_{n}(k+1)}{s_{n}(k)}=\frac{(k+2)^{k+1}}{n(k+1)^{k}}$. Notice this ratio is increasing with $k$, and hence $s_{n}$ is a convex function on $\{1, \ldots, n-2\}$. It follows that the maximum value of $s_{n}$ must occur at one the endpoints, 1 and $n-2$.

Now we show that $s_{n}(n-2) \geqslant s_{n}(1)$ for all $n \geqslant 3$. We can check this explicitly for $n=3,4,5$. When $n \geqslant 6, s_{n}(n-2) / s_{n}(1)=\frac{n}{2}\left(\frac{n-1}{n}\right)^{n-2} \geqslant 3(1 / e)>1$; so the largest syntactic semigroup of $L_{n, k}(\Sigma)$ occurs only at $k=n-2$ for all $n \geqslant 3$. 
Proposition 4 (Reverse). For any regular language $L$ of complexity $n$ with an empty quotient, the reversal has complexity at most $2^{n-1}$. Moreover, the reverse of $L_{n, k}\left(a, b,-,-,-, d_{2}, e\right)$ has complexity $2^{n-1}$ for $n \geqslant 3$ and $1 \leqslant k \leqslant n-2$.

Proof. The first claim is left for the reader to verify. For the second claim, let $\mathcal{D}_{n, k}=\left(Q_{n},\left\{a, b, d_{2}, e\right\}, \delta_{n, k}, 0, F_{n, k}\right)$ denote the DFA $\mathcal{D}_{n, k}\left(a, b,-,-,-, d_{2}, e\right)$ in Definition 2 and let $L_{n, k}=L\left(D_{n, k}\right)$. Construct an NFA $\mathcal{N}$ recognizing the reverse of $L_{n, k}$ by reversing each transition, letting the initial state 0 be the unique final state, and letting the final states in $F_{n, k}$ be the initial states. Applying the subset construction to $\mathcal{N}$ yields a DFA $\mathcal{D}^{R}$ whose states are subsets of $Q_{n-1}$, with initial state $F_{n, k}$ and final states $\left\{U \subseteq Q_{n-1} \mid 0 \in U\right\}$. We show that $\mathcal{D}^{R}$ is minimal, and hence the reverse of $L_{n, k}$ has complexity $2^{n-1}$.

Recall from Lemma 2 that $a$ and $b$ generate all permutations of $E_{n, k}$ and $F_{n, k}$ in $\mathcal{D}_{n, k}$ and, although the transitions are reversed in $\mathcal{D}^{R}$, they still generate all such permutations. Let $u_{1}, u_{2} \in\{a, b\}^{*}$ be such that $u_{1}$ induces $(0, \ldots, n-2-k)$ and $u_{2}$ induces $(n-1-k, \ldots, n-2)$ in $\mathcal{D}^{R}$.

Consider a state $U=\left\{q_{1}, \ldots, q_{h}, n-1-k, \ldots, n-2\right\}$ where $0 \leqslant q_{1}<$ $q_{2}<\cdots<q_{h} \leqslant n-2-k$. If $h=0$, then $U$ is the initial state. When $h \geqslant 1$, $\left\{q_{2}-q_{1}, q_{3}-q_{1}, \ldots, q_{h}-q_{1}, n-1-k, \ldots, n-2\right\} e u_{1}^{q_{1}}=U$. By induction, all such states are reachable.

Now we show that any state $U=\left\{q_{1}, \ldots, q_{h}, p_{1}, \ldots, p_{i}\right\}$ where $0 \leqslant q_{1}<q_{2}<$ $\cdots<q_{h} \leqslant n-2-k$ and $n-1-k \leqslant p_{1}<p_{2}<\cdots<p_{i} \leqslant n-2$ is reachable. If $i=k$, then $U=\left\{q_{1}, \ldots, q_{h}, n-1-k, \ldots, n-2\right\}$ is reachable by the argument above. When $0 \leqslant i<k$, choose $p \in F_{n, k} \backslash U$ and see that $U$ is reached from $U \cup\{p\}$ by $u_{2}^{n-1-p} d_{2} u_{2}^{p-(n-2-k)}$. By induction, every state is reachable.

To prove distinguishability, consider distinct states $U$ and $V$. Choose $q \in$ $U \oplus V$. If $q \in E_{n, k}$, then $U$ and $V$ are distinguished by $u_{1}^{n-1-k-q}$. When $q \in F_{n, k}$, they are distinguished by $u_{2}^{n-1-q} e$. So $\mathcal{D}^{R}$ is minimal.

Proposition 5 (Star). Let $L$ be a regular language with $n \geqslant 2$ quotients, including $k \geqslant 1$ final quotients and one empty quotient. Then $\kappa\left(L^{*}\right) \leqslant 2^{n-2}+$ $2^{n-2-k}+1$. This bound is tight for prefix-convex languages; in particular, the language $\left(L_{n, k}\left(a, b,-,-, d_{1}, d_{2}, e\right)\right)^{*}$ meets this bound for $n \geqslant 3$ and $1 \leqslant k \leqslant n-2$.

Proof. Since $L$ has an empty quotient, let $n-1$ be the empty state of its minimal DFA $\mathcal{D}$. To obtain an $\varepsilon$-NFA for $L^{*}$, we add a new initial state $0^{\prime}$ which is final and has the same transitions as 0 . We then add an $\varepsilon$-transition from every state in $F$ to 0 . Applying the subset construction to this $\varepsilon$-NFA yields a DFA $\mathcal{D}^{\prime}=\left(Q^{\prime}, \Sigma, \delta^{\prime},\left\{0^{\prime}\right\}, F^{\prime}\right)$ recognizing $L^{*}$, in which $Q^{\prime}$ contains non-empty subsets of $Q_{n} \cup\left\{0^{\prime}\right\}$.

Many of the states of $Q^{\prime}$ are unreachable or indistinguishable from other states. Since there is no transition in the $\varepsilon$-NFA to $0^{\prime}$, the only reachable state in $Q^{\prime}$ containing $0^{\prime}$ is $\left\{0^{\prime}\right\}$. As well, any reachable final state $U \neq\left\{0^{\prime}\right\}$ must contain 0 because of the $\varepsilon$-transitions. Finally, for any $U \in Q^{\prime}$, we have $U \in F^{\prime}$ if and only if $U \cup\{n-1\} \in F^{\prime}$, and since $\delta^{\prime}(U \cup\{n-1\}, w)=\delta^{\prime}(U, w) \cup\{n-1\}$ for all $w \in \Sigma^{*}$, the states $U$ and $U \cup\{n-1\}$ are equivalent in $D^{\prime}$. 
Hence $\mathcal{D}^{\prime}$ is equivalent to a DFA with the states $\left\{\left\{0^{\prime}\right\}\right\} \cup\left\{U \subseteq Q_{n-1} \mid U \cap F=\right.$ $\emptyset\} \cup\left\{U \subseteq Q_{n-1} \mid 0 \in U\right.$ and $\left.U \cap F \neq \emptyset\right\}$. This DFA has $1+2^{n-1-k}+\left(2^{n-2}-\right.$ $\left.2^{n-2-k}\right)=2^{n-2}+2^{n-2-k}+1$ states. Thus, $\kappa\left(L^{*}\right) \leqslant 2^{n-2}+2^{n-2-k}+1$.

This bound applies when $L$ is a prefix-convex language and $n \geqslant 3$. By Lemma $1, L$ is either a right ideal or has an empty state. If $L$ is a right ideal, then $\kappa\left(L^{*}\right) \leqslant n+1$, which is at most $2^{n-2}+2^{n-2-k}+1$ for $n \geqslant 3$.

For the last claim, let $\mathcal{D}_{n, k}\left(a, b,-,-, d_{1}, d_{2}, e\right)$ of Definition 2 be denoted by $\mathcal{D}_{n, k}=\left(Q_{n},\left\{a, b, d_{1}, d_{2}, e\right\}, \delta_{n, k}, 0, F_{n, k}\right)$ and let $L_{n, k}=L\left(D_{n, k}\right)$. We apply the same construction and reduction as before to obtain a DFA $\mathcal{D}_{n, k}^{\prime}$ recognizing $L_{n, k}^{*}$ with states $Q^{\prime}=\left\{\left\{0^{\prime}\right\}\right\} \cup\left\{U \subseteq E_{n, k}\right\} \cup\left\{U \subseteq Q_{n-1} \mid 0 \in U\right.$ and $\left.U \cap F_{n, k} \neq \emptyset\right\}$. We show that the states of $Q^{\prime}$ are reachable and pairwise distinguishable.

By Lemma 2, $a$ and $b$ generate all permutations of $E_{n, k}$ and $F_{n, k}$ in $\mathcal{D}_{n, k}$. Choose $u_{1}, u_{2} \in\{a, b\}^{*}$ such that $u_{1}$ induces $(0, \ldots, n-2-k)$ and $u_{2}$ induces $(n-1-k, \ldots, n-2)$ in $\mathcal{D}_{n, k}$.

For reachability, we consider three cases. (1) State $\left\{0^{\prime}\right\}$ is reachable by $\varepsilon$. (2) Let $U \subseteq E_{n, k}$. For any $q \in E_{n, k}$, we can reach $U \backslash\{q\}$ by $u_{1}^{n-2-k-q} d_{1} u_{1}^{q}$; hence if $U$ is reachable, then every subset of $U$ is reachable. Observe that state $E_{n, k}$ is reachable by $e u_{1}^{n-2-k} d_{2}^{k}$, and we can reach any subset of this state. Therefore, all non-final states are reachable. (3) If $U \cap F_{n, k} \neq \emptyset$, then $U=$ $\left\{0, q_{1}, q_{2}, \ldots, q_{h}, r_{1}, \ldots, r_{i}\right\}$ where $0<q_{1}<\cdots<q_{h} \leqslant n-2-k$ and $n-1-k \leqslant$ $r_{1}<\cdots<r_{i}<n-1$ and $i \geqslant 1$. We prove that $U$ is reachable by induction on $i$. If $i=0$, then $U$ is reachable by (2). For any $i \geqslant 1$, we can reach $U$ from $\left\{0, q_{1}, \ldots, q_{h}, r_{2}-\left(r_{1}-(n-1-k)\right), \ldots, r_{i}-\left(r_{1}-(n-1-k)\right)\right\}$ by $e u_{2}^{r_{1}-(n-1-k)}$. Therefore, all states of this form are reachable.

Now we show that the states are pairwise distinguishable. (1) The initial state $\left\{0^{\prime}\right\}$ is distinguishable from any other final state $U$ since $\left\{0^{\prime}\right\} u_{1}$ is non-final and $U u_{1}$ is final. (2) If $U$ and $V$ are distinct subsets of $E_{n, k}$, then there is some $q \in U \oplus V$. We distinguish $U$ and $V$ by $u_{1}^{n-1-k-q} e$. (3) If $U$ and $V$ are distinct and final and neither one is $\left\{0^{\prime}\right\}$, then there is some $q \in U \oplus V$. If $q \in E_{n, k}$, then $U d_{2}^{k}=U \backslash F_{n, k}$ and $V d_{2}^{k}=V \backslash F_{n, k}$ are distinct, non-final states as in (2). Otherwise, $q \in F_{n, k}$ and we distinguish $U$ and $V$ by $u_{2}^{n-1-q} d_{2}^{k-1}$.

\section{Conclusions}

The bounds for prefix-convex languages (see also [8]) are summarized in Table 1 . The largest bounds are shown in boldface type, and they are reached either in the class of right-ideal languages or the class of proper languages. Recall that for regular languages we have the following results: semigroup $n^{n}$, reverse $2^{n}$, star $2^{n-1}+2^{n-2}$, product $m 2^{n}-2^{n-1}$, boolean operations $m n$.

\section{References}

1. T. Ang and J. A. Brzozowski. Languages convex with respect to binary relations, and their closure properties. Acta Cybernet., 19(2):445-464, 2009. 
Table 1. Complexities of prefix-convex languages

\begin{tabular}{|c||c|c|c|c|}
\hline & Right-Ideal & Prefix-Closed & Prefix-Free & Proper \\
\hline \hline SeGr & $\mathbf{n}^{\mathbf{n}-\mathbf{1}}$ & $\mathbf{n}^{\mathbf{n}-\mathbf{1}}$ & $n^{n-2}$ & $n^{n-1-k}(k+1)^{k}$ \\
\hline Rev & $\mathbf{2}^{\mathbf{n}-\mathbf{1}}$ & $\mathbf{2}^{\mathbf{n - 1}}$ & $2^{n-2}+1$ & $\mathbf{2}^{\mathbf{n}-\mathbf{1}}$ \\
\hline Star & $n+1$ & $2^{n-2}+1$ & $n$ & $\mathbf{2}^{\mathbf{n - 2}}+\mathbf{2}^{\mathbf{n}-\mathbf{2}-\mathbf{k}}+\mathbf{1}$ \\
\hline Prod & $m+2^{n-2}$ & $(m+1) 2^{n-2}$ & $m+n-2$ & $\mathbf{m}-\mathbf{1}-\mathbf{j}+\mathbf{j} \mathbf{2}^{\mathbf{n - 2}}+\mathbf{2}^{\mathbf{n - 1}}$ \\
\hline$\cup$ & $m n-(m+n-2)$ & $\mathbf{m n}$ & $m n-2$ & $\mathbf{m n}$ \\
\hline$\oplus$ & $\mathbf{m n}$ & $\mathbf{m n}$ & $m n-2$ & $\mathbf{m n}$ \\
\hline$\backslash$ & $\mathbf{m n}-(\mathbf{m}-\mathbf{1})$ & $\mathbf{m n}-(\mathbf{n}-\mathbf{1})$ & $m n-(m+2 n-4)$ & $\mathbf{m n}-(\mathbf{n}-\mathbf{1})$ \\
\hline$\cap$ & $\mathbf{m n}$ & $m n-(m+n-2)$ & $m n-2(m+n-3)$ & $m n-(m+n-2)$ \\
\hline
\end{tabular}

2. J. Berstel, D. Perrin, and C. Reutenauer. Codes and Automata (Encyclopedia of Mathematics and its Applications). Cambridge University Press, 2010.

3. J. A. Brzozowski. Quotient complexity of regular languages. J. Autom. Lang. Comb., 15(1/2):71-89, 2010.

4. J. A. Brzozowski. In search of the most complex regular languages. Int. J. Found. Comput. Sci, 24(6):691-708, 2013.

5. J. A. Brzozowski, S. Davies, and B. Y. V. Liu. Most complex regular ideal languages. Discrete Math. Theoret. Comput. Sc., 18(3), 2016. Paper \#15.

6. J. A. Brzozowski, G. Jirásková, and C. Zou. Quotient complexity of closed languages. Theory Comput. Syst., 54:277-292, 2014.

7. J. A. Brzozowski and C. Sinnamon. Complexity of prefix-convex regular languages. http://arxiv.org/abs/1605.06697, 2016.

8. J. A. Brzozowski and C. Sinnamon. Complexity of right-ideal, prefix-closed, and prefix-free regular languages. Acta Cybernet., 2017. To appear.

9. J. A. Brzozowski and H. Tamm. Theory of átomata. Theoret. Comput. Sci., 539:13-27, 2014.

10. J. A. Brzozowski and Y. Ye. Syntactic complexity of ideal and closed languages. In G. Mauri and A. Leporati, editors, DLT 2011, volume 6795 of $L N C S$, pages 117-128. Springer Berlin / Heidelberg, 2011.

11. M. Crochemore and C. Hancart. Automata for pattern matching. In G. Rozenberg and A. Salomaa, editors, Handbook of Formal Languages, volume 2, pages 399-462. Springer, 1997.

12. M. Holzer and B. König. On deterministic finite automata and syntactic monoid size. Theoret. Comput. Sci., 327(3):319-347, 2004.

13. S. Iván. Complexity of atoms, combinatorially. Inform. Process. Lett., 116(5):356$360,2016$.

14. B. Krawetz, J. Lawrence, and J. Shallit. State complexity and the monoid of transformations of a finite set. In M. Domaratzki and et al., editors, CIAA 2005, volume 3317 of $L N C S$, pages 213-224. Springer Berlin / Heidelberg, 2005.

15. A. N. Maslov. Estimates of the number of states of finite automata. Dokl. Akad. Nauk SSSR, 194:1266-1268 (Russian)., 1970. English translation: Soviet Math. Dokl. 11 (1970) 1373-1375.

16. G. Thierrin. Convex languages. In M. Nivat, editor, Automata, Languages and Programming, pages 481-492. North-Holland, 1973.

17. S. Yu. State complexity of regular languages. J. Autom. Lang. Comb., 6:221-234, 2001.

18. S. Yu, Q. Zhuang, and K. Salomaa. The state complexities of some basic operations on regular languages. Theoret. Comput. Sci., 125(2):315-328, 1994. 